\title{
Recombination-line Abundances for the Third-Row Element, Magnesium
}

\author{
M. J. Barlow ${ }^{1}$, X.-W. Liu ${ }^{1}$, D. Péquignot ${ }^{2}$, P. J. Storey ${ }^{1}$, \\ Y. G. Tsamis ${ }^{1}$, C. Morisset ${ }^{3}$ \\ ${ }^{1}$ Department of Physics and Astronomy, University College London, \\ Gower Street, London, WC1E 6BT, U.K. \\ ${ }^{2}$ Observatoire de Paris-Meudon, France \\ ${ }^{3}$ Institut d'Astrophysique de Marseille, France
}

\section{Introduction}

We have obtained optical recombination-line abundances for the third-row element magnesium in ten planetary nebulae. The nebulae in our sample include four (NGC 7009, NGC 6153, M 2-36 and M 1-42) for which we have previously found very large enhancements, relative to solar, in the recombination line abundances of the second-row elements carbon, nitrogen, oxygen and neon (Liu et al. 1995, Liu et al. 2000, Liu et al. 2001). Nebular temperature fluctuations appear unable to account for these effects. However, models that invoke high-density clumps, particularly clumps which are hydrogen-deficient, appear more viable as an explanation for the high recombination line abundances that are observed (see Liu et al. 2000 for more details). While it may be possible to appeal to astrophysical nucleosynthetic processes to enhance the CNONe second-row elements in AGB stars, such effects are not expected to modify the abundances of third-row elements such as magnesium. We have therefore measured and dereddened the intensities of the $4481 \AA 4 \mathrm{f}-3 \mathrm{~d}$ line of $\mathrm{Mg}$ II, relative to $\mathrm{H} \beta$, in order to investigate whether magnesium recombination line abundances are enhanced or not.

\section{Analysis}

$\mathrm{Mg}^{2+}$ occupies an unusually large ionization potential interval, from $15 \mathrm{eV}$ to $80 \mathrm{eV}$, so that it is the dominant ion stage of magnesium in most photoionized nebulae. Photoionization models for a a wide range of nebular excitation classes show that $\mathrm{Mg}^{2+}$ accounts for $90 \%$ or more of magnesium in all but the highest excitation nebulae. In addition, its recombination product, $\mathrm{Mg}$ II, has a particularly simple spectrum (like C II), concentrated in a relatively small number of transitions. As a result, the $\mathrm{Mg}$ II $4 \mathrm{f}-3 \mathrm{~d}$ line at $4481.21 \AA$ is the strongest and easiest to measure optical recombination line from any third-row ion. To simplify the analysis, we have determined $\mathrm{O}^{2+}$ abundances from the $4 \mathrm{f}-3 \mathrm{~d}$ line of $\mathrm{O}$ II at $4089.29 \AA$, in order to compare our derived $\mathrm{Mg}^{2+}$ abundances with those of $\mathrm{O}^{2+}$. 
To derive $\mathrm{O}^{2+} / \mathrm{H}^{+}$and $\mathrm{Mg}^{2+} / \mathrm{H}^{+}$abundances for the 13 nebulae in our sample, the $\mathrm{H} \beta$ intensities were first corrected for the contribution by the He II 8-4 transition, using the dereddened He II 4-3 $4686 \AA$ intensities and the recombination theory predictions of Storey and Hummer (1995). The derived abundance ratios have a very weak dependence on the adopted nebular electron temperature. We adopted [O III] forbidden line temperatures in most cases, although in the case of the four 'super-metal-rich' nebulae we adopted their hydrogen Balmer jump temperatures for our recombination line analysis.

The four nebulae that had previously been found by us to exhibit very enhanced second-row recombination line abundances show $\mathrm{O}^{2+} / \mathrm{H}^{+}$abundances, using the O II $4089.29 \AA$ line, that are enhanced by factors of 4.8 (NGC 7009), 8.6 (M 2-36), 10.0 (NGC 6153) and 12.1 (M 1-42) relative to the solar $\mathrm{O} / \mathrm{H}$ ratio, while the six 'average' PNe (NGC's 3242, 3918, 5315, 5882 and 6543, plus IC 4191), show recombination line $\mathrm{O}^{2+} / \mathrm{H}^{+}$abundances that are enhanced by factors of between 1.7 and 3.2 above the solar value $\left(4.90 \times 10^{-4}\right.$, Allende Prieto et al. 2001; they used high S/N spectra of the weak [O I] $6300 \AA$ absorption line in the solar spectrum to revise the solar oxygen abundance downwards by 0.2 dex, to a value which is now in agreement with the mean PN forbidden-line oxygen abundance determined by Kingsburgh \& Barlow 1994). By way of contrast, the magnesium recombination line abundances of all ten $\mathrm{PNe}$ in our sample are found to display a remarkably narrow range: $(4.09 \pm 0.94) \times 10^{-5}$ for the four $\mathrm{PNe}$ with large second-row element enhancements, and $(2.72 \pm 0.45) \times 10^{-5}$ for the middle group of six 'average' PNe. These values can be compared to the solar $\mathrm{Mg} / \mathrm{H}$ ratio of $3.45 \times 10^{-5}$ (Holweger 2001). The gas-phase abundance of magnesium in the interstellar medium is depleted by only $30 \%$ relative to solar values (Sofia \& Meyer 2001) - in the harsh nebular environments of PNe the degree of depletion onto dust dust grains is expected to be even smaller. The narrow range and the lack of large enhancements over solar in the abundance of this third-row element, compared to the wide range of enhancements found for oxygen and for other second-row elements in the same sample, suggests that the second-row recombination line abundance enhancements are due to astrophysical effects (e.g. H-deficient nebular knots) rather than some unrecognised atomic process, since the latter might be expected to affect ions of the 3rd-row element magnesium to the same degree as 2nd-row ions.

\section{References}

Holweger H., 2001, in 'Solar and Galactic Composition', ed. R. F. Wimmer-Schweingruber. AIP Conf. Proc. vol. 598, p.23

Kingsburgh R. L., \& Barlow M. J., 1994, MNRAS, 271, 257

Liu X.-W., Storey P. J., Barlow M. J., Clegg, R. E. S., MNRAS, 1995, 272, 369

Liu X.-W., et al., 2000, MNRAS, 312, 585

Liu X.-W., et al., 2001, MNRAS, 327, 141

Allende Prieto C., Lambert D. L., Asplund M., 2001, ApJ, 556, L63

Sofia U. J., \& Meyer D. M., 2001, ApJ, 554, L221

Storey P. J., \& Hummer D. G., 1995, MNRAS, 272, 41 